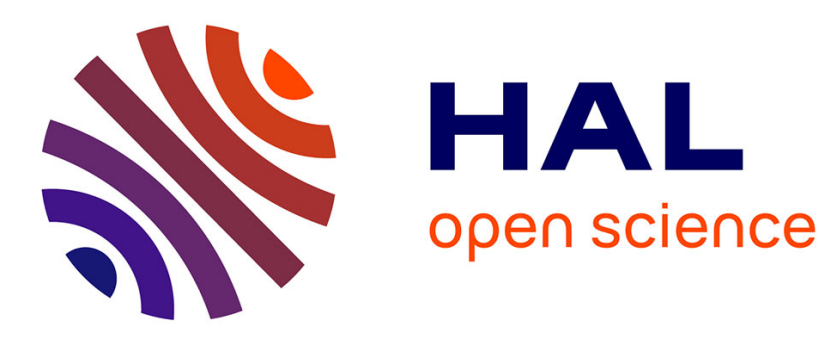

\title{
Linear Time-Varying (Max,+) Representation of Conflicting Timed Event Graphs
}

Boussad Addad, Saïd Amari, Jean-Jacques Lesage

\section{To cite this version:}

Boussad Addad, Saïd Amari, Jean-Jacques Lesage. Linear Time-Varying (Max,+) Representation of Conflicting Timed Event Graphs. 10th IFAC Workshop on Discrete Event Systems, Aug 2010, Berlin, Germany. pp.310-315. hal-00514880

\section{HAL Id: hal-00514880 \\ https://hal.science/hal-00514880}

Submitted on 3 Sep 2010

HAL is a multi-disciplinary open access archive for the deposit and dissemination of scientific research documents, whether they are published or not. The documents may come from teaching and research institutions in France or abroad, or from public or private research centers.
L'archive ouverte pluridisciplinaire HAL, est destinée au dépôt et à la diffusion de documents scientifiques de niveau recherche, publiés ou non, émanant des établissements d'enseignement et de recherche français ou étrangers, des laboratoires publics ou privés. 


\title{
Linear Time-Varying (Max,+) Representation of Conflicting Timed Event Graphs
}

\author{
ADDAD, B.*, AMARI, S.*, LESAGE, J-J.* \\ *Automated Production Research Laboratory (LURPA), 61 av du President Wilson, Cachan 94235 France, \\ (Tel: (+33) 01474027 62; e-mail: Boussad.addad@lurpa.ens-cachan.fr, said.amari@lurpa.ens-cachan.fr, Jean- \\ jacques.lesage@lurpa.ens-cachan.fr).
}

\begin{abstract}
Timed Event Graphs (TEGs) are a specific class of Petri nets that have been thoroughly studied given their useful linear state representation in $(\mathrm{Max},+)$ algebra. Unfortunately, TEGs are generally not suitable for modeling systems displaying resources sharing (or conflicts). In this paper, we show that if a system with conflicts is modeled using an extended class of TEGs: Conflicting Timed Event Graphs (CTEGs), then it is quite possible to obtain an equivalent $(\mathrm{Max},+)$ representation. More precisely, we prove that the evolution of a CTEG satisfies linear time-varying $(\mathrm{Max},+)$ equations. In case of cyclic CTEGs, which are a natural model of many repetitive systems, we provide a standard timeinvariant $(\mathrm{Max},+)$ representation. Finally, a practical example (a Jobshop) is used for illustration to exhibit the interest of this investigation.
\end{abstract}

Keywords: Timed Event Graph, (Max,+) algebra, Conflict, Linear-Time Varying Systems, Repetitive Systems.

\section{INTRODUCTION}

Petri nets are a powerful tool for discrete events systems modeling and analysis. They are often used to represent phenomena like synchronization, parallelism and concurrency (Murata, 1989). Their domain of application is very large, including manufacturing systems, communication systems, transportation, etc. Many classes of Petri nets with more or less elaborated semantics are used according to the context of the study. Generally speaking, the more their structure and semantics are elaborated the more complex is their study. The relatively simple class of Petri nets called Timed Event Graphs (TEGs) is then the most investigated one. Indeed, TEGs are easily represented in the form of linear equations in $(\mathrm{Max},+)$ algebra provided that the places and the transitions be overtaking free (FIFO places and FIFO transitions as defined in Baccelli et al. (1992). This linear $(\mathrm{Max},+)$ form being very similar to the state representation of the classical discrete linear systems, the main related results are mostly straightforwardly applied (Cohen et al. 1999). Unfortunately, TEGs are Petri nets with places displaying at most one upstream transition and one downstream transition. Thus, they are not suitable for modeling systems with resources sharing or conflicts. Note nonetheless that some particular systems involving shared resources (e.g. flowshops), can be modelled using TEGs with time-varying parameters (Lahaye et al., 2004). The authors had however to change the usual FIFO rule to prevent tokens from overtaking and finally to get to linear $(\mathrm{Max},+)$ equations. Such a consideration is possible in some cases but it is unfortunately not always the case. In literature, a number of other efforts have been undertaken to tackle differently the problem of conflicts. The authors in Hillion et al. (1989) investigated the problem of repetitive systems i.e. with a cyclic allocation of the shared resources. They proposed to transform the original Petri net with conflicts into a TEG, the (Max, + ) representation of this latter being easily obtained. In Gaubert et al. (1999), an algebraic modeling, based on the heaps-ofpieces theory and $(\mathrm{Max},+)$ automata, is provided for safe Petri nets. For the case of Free Choice Petri nets, a very complete analytic study is proposed in Baccelli et al. (1996). The case of processes that switch between different functioning modes is investigated in Van Den Boom et al. (2006) using switching $(\mathrm{Max},+)$ linear systems. We can also quote Correïa et al. (2009) where local $(\mathrm{Max},+)$ equations are written without taking into account the conflicts. A constraint (inequality) is then added to represent only the admissible evolutions of the global system. In Nait et al. (2006), a method introducing the concept of virtual firing of transitions is proposed for a transportation system. More recently (Boutin et al., 2009), an approach based on the dioid of intervals is used to represent extreme behaviors of a manufacturing system (with shared resources i.e. conflicts context) and provide the bounds of its production rate.

In this paper, we propose a novel approach to model a large category of systems involving shared resources. The main idea of our work has been first introduced in Addad et al. (2010) but has been limited to systems with one shared resource. In this paper, we present a generalization of this approach and consider systems with multiple resources. The purpose of the study is to extend the use of $(\mathrm{Max},+)$ algebra to a class of Petri nets larger than TEGs: Conflicting Timed Event Graphs (CTEGs). Moreover, some of the previously exposed hypotheses in the existing works are relaxed:

i) the TEGs that constitute the CTEG are not necessarily safe (they, by the way, display input transitions). 
ii) the conflicting transitions can have more than one upstream place (unlike Free Choice Petri nets).

iii) resources allocation policy is not necessarily cyclic.

The remainder of this paper is organized as follows: Section 2 recalls some basic notions about TEGs and their linear $(\mathrm{Max},+)$ representation. Section 3 is dedicated to the study of CTEGs in $(\mathrm{Max},+)$ algebra: Section 3.1 introduces some definitions and notations with regard to CTEGs and Section 3.2 presents their modeling using $(\mathrm{Max},+)$ equations. Thereafter, a linear time-varying reformulation is provided in Section 4. Hence, a standard time-invariant linear state form is provided for cyclic CTEGs in Section 5. Finally, Section 6 concludes this paper with some outlooks for future work.

\section{LINEAR $(\mathrm{MAX},+)$ EQUATIONS OF TEGs}

In this section, we recall the linear $(\mathrm{Max},+)$ representation of TEGs that will be useful in the sequel of this paper. Note that a mix of timed places and timed transitions is considered in our study and if their delays are not précised, then they are null. Let's consider the simple example below (t-timed):

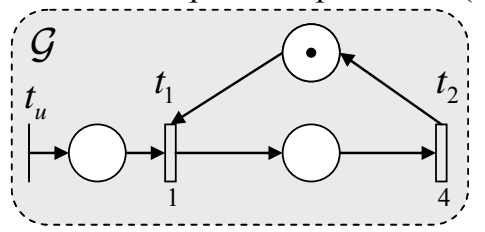

Fig. 1. Example of TEG.

Let $x_{i}(k)$ (resp. $u(k)$ ) be the date of firing transition $t_{i}$ (resp. $t_{u}$ ) for the $k^{\text {th }}$ time. If firing is performed at maximal speed (as soon as possible), we can write the equations:

$\left\{\begin{array}{l}x_{1}(k)=1+\max \left(u(k), x_{2}(k-1)\right) \\ x_{2}(k)=4+x_{1}(k)\end{array}\right.$

The equations above can be rewritten using $(\mathrm{Max},+)$ algebra operators: the maximum noted $\oplus$ and addition noted $\otimes$. These operators are defined on the set $\mathbb{R}_{\max }=\mathbb{R} \cup\{-\infty\}$ and have respectively $\varepsilon=-\infty$ and $e=0$ as identity elements. Equations (1) become:

$\left\{\begin{array}{l}x_{1}(k)=1 \otimes u(k) \oplus 1 \otimes x_{2}(k-1) \\ x_{2}(k)=4 \otimes x_{1}(k)\end{array}\right.$

Using a matrix notation, we finally get to:

$X(k)=\hat{A} \otimes X(k) \oplus \hat{A}^{\prime} \otimes X(k-1) \oplus \hat{B} \otimes U(k)$

with: $X=\left(\begin{array}{ll}x_{1} & x_{2}\end{array}\right)^{t}, U=u, \hat{A}=\left(\begin{array}{ll}\varepsilon & \varepsilon \\ 4 & \varepsilon\end{array}\right), \hat{A}^{\prime}=\left(\begin{array}{ll}\varepsilon & 1 \\ \varepsilon & \varepsilon\end{array}\right), \hat{B}=\left(\begin{array}{l}1 \\ \varepsilon\end{array}\right)$.

Equation (3) can also be brought to an explicit form thanks to the following theorem:

Theorem 2.1 (Baccelli et al. 1992): The minimal solution of equation: $X=A \otimes X \oplus B$ with $A \in \mathbb{R}_{\max }^{n \times n}, B \in \mathbb{R}_{\max }^{n \times m}$ is: $X=A^{*} \otimes B$ with $A^{*}=\underset{i \in \mathbb{N}}{\oplus} A^{i}$ being the Kleene star of $A$.

By applying the previous theorem to equations (3), we get to the following explicit form:

$X(k)=A \otimes X(k-1) \oplus B \otimes U(k)$ with: $\hat{A}^{*}=\left(\begin{array}{ll}e & \varepsilon \\ 4 & e\end{array}\right), A=\hat{A}^{*} \cdot \hat{A}^{\prime}=\left(\begin{array}{ll}\varepsilon & 1 \\ \varepsilon & 5\end{array}\right)$, and $B=\hat{A}^{*} \cdot \hat{B}=\left(\begin{array}{l}1 \\ 5\end{array}\right)$.

Remark 2.1: Equation (3) and its explicit form (4) feature every TEG. It is a standard state representation like the classical representation of linear systems. Thus, it is widely used alike to solve many problems of performance evaluation or control synthesis (Cohen, et al. 1999).

\section{MODELING CTEGs IN (MAX,+) ALGEBRA}

\subsection{Definitions and notations}

A net of Conflicting Timed Event Graphs or CTEG is a set of TEGs noted $\mathcal{G}=\left\{\mathcal{G}_{1}, \mathcal{G}_{2}, \cdots, \mathcal{G}_{N}\right\}$ (e.g. products, jobs, users...) connected to each other by a set of conflict places (e.g. shared resources) noted $\tilde{R}=\left\{\tilde{p}_{1}, \tilde{p}_{2}, \cdots, \tilde{p}_{M}\right\}$. Each timed event graph $\mathcal{G}_{i}$ is connected to a subset of conflict places (or resources) noted $\tilde{R}_{i}\left(\tilde{R}_{i} \subseteq \tilde{R}\right)$. With place $\tilde{p}_{j} \in \tilde{R}_{i}$, we get the generic structure of CTEG on Fig. 2(a): (a)

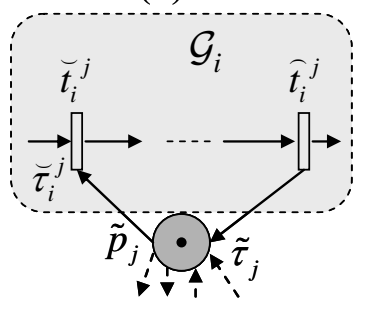

(b)

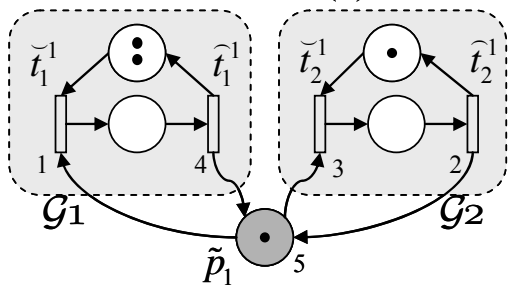

Fig. 2. (a) Generic structure of a CTEG $\left(\tilde{p}_{j} \in \tilde{R}_{i}\right)$, (b) example of CTEG (one conflict place and two TEGs).

For the sake of simplicity, we will adopt the following hypotheses and notations:

- H1) the unique transition of $\mathcal{G}_{i}$ upstream place $\tilde{p}_{j}$ is noted $\bar{t}_{i}^{j}$ and the unique downstream transition is noted $\breve{t}_{i}^{j}$ (Fig. 2(a)). We suppose also, without loss of generality (since we can always get to this case by duplicating the transition $\breve{t}_{i}^{j}$ ) that $\breve{t}_{i}^{j}$ has a unique upstream place of conflict $\tilde{p}_{j}$.

- H2) a shared resource is used by at most one user at a time. So, in terms of Petri nets, each circuit $\breve{t}_{i}^{j} \cdots{\bar{t}_{i}^{j}}^{j} \tilde{p}_{j} \breve{t}_{i}^{j}$ is safe and the sum of markings of all the circuits involving a place $\tilde{p}_{j}$ is exactly equal to one. A resource is indeed either idle or being used by one among the $N$ TEGs.

- H3) the holding time associated to place $\tilde{p}_{j}$ is denoted $\tilde{\tau}_{j}$ and the firing time of $\breve{t}_{i}^{j}$ is denoted $\breve{\tau}_{i}^{j}$ (see Fig. 2(a)).

- H4) each TEG $\mathcal{G}_{i}$ is alive, represented by its state form: $X_{i}\left(k_{i}\right)=\hat{A}_{i} \otimes X_{i}\left(k_{i}\right) \oplus \hat{A}_{i}^{\prime} \otimes X_{i}\left(k_{i}-1\right) \oplus \hat{B}_{i} \otimes U_{i}\left(k_{i}\right)$ where: $X_{i}=\left(\begin{array}{llll}x_{i}^{1} & x_{i}^{2} & \cdots & x_{i}^{n_{i}}\end{array}\right)^{t}$ is an $n_{i} \times 1$ state vector and 
$U_{i}=\left(\begin{array}{llll}u_{i}^{1} & u_{i}^{2} & \cdots & u_{i}^{m_{i}}\end{array}\right)^{t}$ is an $m_{i} \times 1$ input vector. In the remaining of this study, the $i^{\text {th }}$ row of a matrix $A$ is denoted $A(i,:)$ whereas the intersection element of the $i^{\text {th }}$ row and the $j^{\text {th }}$ column is denoted $A(i, j)$.

\subsection{CTEG modeling using (Max,+) equations}

In studying TEGs, daters are usually associated to the transitions. In our approach however, we also associate a dater to the token of every place of conflict $\tilde{p}_{j} \in \tilde{R}$. The date of availability of this token for the $l_{j}^{\text {th }}$ time is denoted $\tilde{\psi}_{j}\left(l_{j}\right)$. Intuitively, this is the date of availability of the shared resource $\tilde{p}_{j}$ after being used $l_{j}$ times.

Let's suppose that every transition of TEG $\mathcal{G}_{i}$ has already fired $\left(k_{i}-1\right)$ times. To fire for the $k_{i}^{\text {th }}$ time, each transition needs all its upstream places to have at least one available token. So, a conflicting transition $\breve{t}_{i}^{j}$ needs the token of its upstream place of conflict $\tilde{p}_{j}$ to be available too. Suppose that the token of this place is attributed effectively to $\mathcal{G}_{i}$ when it is available for the $l_{j}^{\text {th }}$ time. If $x_{i}^{j}$ is the dater of vector $X_{i}$ associated to transition $\breve{t}_{i}^{j}$, then we can write:

$$
\begin{aligned}
& X_{i}^{j}\left(k_{i}\right)=\hat{A}_{i}(j,:) \otimes X_{i}\left(k_{i}\right) \oplus \hat{A}_{i}^{\prime}(j,:) \otimes X_{i}\left(k_{i}-1\right) \\
& \oplus \hat{B}_{i}(j,:) \otimes U_{i}\left(k_{i}\right) \oplus \tilde{\tau}_{i}^{j} \otimes \tilde{\psi}_{j}\left(l_{j}\right)
\end{aligned}
$$

Equation (6) is written for every dater associated to a transition downstream a place of conflict belonging to $\tilde{R}_{i}$. The other daters $x_{i}^{j^{\prime}}$ however, whose transitions are not downstream places of conflict, are unchanged in (5) since they do not depend directly on $\tilde{\psi}_{j}\left(l_{j}\right)$. So, we have:

$$
\begin{aligned}
& x_{i}^{j^{\prime}}\left(k_{i}\right)=\hat{A}_{i}\left(j^{\prime},:\right) \otimes X_{i}\left(k_{i}\right) \oplus \hat{A}_{i}^{\prime}\left(j^{\prime},:\right) \otimes X_{i}\left(k_{i}-1\right) \\
& \oplus \hat{B}_{i}\left(j^{\prime},::\right) \otimes U_{i}\left(k_{i}\right)
\end{aligned}
$$

By combining (6) and (7), we get to the new state form:

$$
\begin{aligned}
& X_{i}\left(k_{i}\right)=\hat{A}_{i} \otimes X_{i}\left(k_{i}\right) \oplus \hat{A}_{i}^{\prime} \otimes X_{i}\left(k_{i}-1\right) \\
& \oplus \hat{B}_{i} \otimes U_{i}\left(k_{i}\right) \oplus \bigoplus_{\tilde{p}_{j} \in \tilde{R}_{i}} \hat{F}_{i j} \otimes \tilde{\psi}_{j}\left(l_{j}\right)
\end{aligned}
$$

with all the involved matrices and vectors are exactly the same as in (5). The only new elements are the availability dates $\tilde{\psi}_{j}\left(l_{j}\right)$ defined previously and matrices $\hat{F}_{i j}$. We can note that the components of the $n_{i} \times 1$ matrix $\hat{F}_{i j}$ are all null (equal to $\varepsilon$ ) except for the $j^{\text {th }}$ one (equal to $\bar{\tau}_{i}^{j}$ ).

By applying Theorem 2.1 to (8), we finally obtain:

$$
X_{i}\left(k_{i}\right)=A_{i} \otimes X_{i}\left(k_{i}-1\right) \oplus B_{i} \otimes U_{i}\left(k_{i}\right) \oplus \underset{\tilde{p}_{j} \in \tilde{R}_{i}}{\bigoplus} F_{i j} \otimes \tilde{\psi}_{j}\left(l_{j}\right)
$$

where: $A_{i}=\hat{A}_{i}^{*} \otimes \hat{A}_{i}^{\prime}, B_{i}=\hat{A}_{i}^{*} \otimes \hat{B}_{i}$ and $F_{i j}=\hat{A}_{i}^{*} \otimes \hat{F}_{i j}$.
On the other hand, since the token of $\tilde{p}_{j}$ is consumed by $\mathcal{G}_{i}$ (or $\breve{t}_{i}^{j}$ ) when it is available for the $l_{j}^{\text {th }}$ time, then it is available for the $\left(l_{j}+1\right)^{\text {th }}$ time by the firing of transition ${\bar{t}_{i}^{j}}^{j}$ for the $k_{i}^{\text {th }}$ time (recall from hypothesis $H 2$ that circuit $\breve{t}_{i}^{j} \cdots \widehat{t}_{i}^{j} \tilde{p}_{j} \breve{t}_{i}^{j}$ is safe). Let $x_{i}^{j "}$ be the component of $X_{i}$ associated to transition ${\bar{t}_{i}}^{j}$. Thus, we can write:

$\tilde{\psi}_{j}\left(l_{j}+1\right)=\tilde{\tau}_{j} \otimes x_{i}^{j "}\left(k_{i}\right)$

This equation can be rewritten as:

$\tilde{\psi}_{j}\left(l_{j}+1\right)=G_{i j} \otimes X_{i}\left(k_{i}\right)$

with $G_{i j}$ is a $1 \times n_{i}$ matrix whose all components are null except for one (equal to $\tilde{\tau}_{j}$ ) corresponding to transition ${t_{i}}_{i}^{j}$. To sum up, if the $l_{j}^{\text {th }}$ token of $\tilde{p}_{j}$ contributes to firing the transitions of TEG $\mathcal{G}_{i}$ for the $k_{i}^{\text {th }}$ time (note that because of resources invariance we have: $\left.l_{j}=\sum_{\tilde{p}_{j} \in \tilde{R}_{i}}\left(k_{i}-1\right)\right)$, then the following recurrent $(\max ,+)$ equations are verified:

$$
\left\{\begin{array}{l}
X_{i}\left(k_{i}\right)=A_{i} \otimes X_{i}\left(k_{i}-1\right) \oplus B_{i} \otimes U_{i}\left(k_{i}\right) \oplus \bigoplus_{\tilde{p}_{j} \in \tilde{R}_{i}} F_{i j} \otimes \tilde{\psi}_{j}\left(l_{j}\right) \\
\tilde{\psi}_{j}\left(l_{j}+1\right)=G_{i j} \otimes X_{i}\left(k_{i}\right) \quad \text { for all } \tilde{p}_{j} \in \tilde{R}_{i}
\end{array}\right.
$$

As can be seen, equations (12) include a parameter, $l_{j}$ in $\tilde{\psi}_{j}\left(l_{j}\right)$, that defines entirely the policy of resources allocation. Hence, these equations represent all the admissible evolutions of CTEGs. All the involved elements are systematically calculated according to the method explained before. These equations are important and will be the cornerstone of all the results provided later in this paper.

Example 3.1: to illustrate the previous method, let us consider the following example (note that if the holding time or firing time is not mentioned, then it is equal to 0 ):

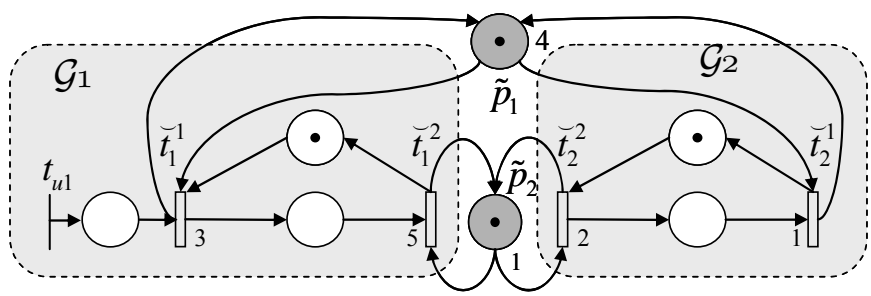

Fig. 3. A CTEG with two conflict places shared by two TEGs (note that in this example $\widehat{t}_{i}^{j} \equiv \breve{t}_{i}^{j} \forall i, j$ ).

By assigning daters $x_{1}^{1}, x_{1}^{2}, x_{2}^{1}$ and $x_{2}^{2}$ to transitions $\breve{t}_{1}^{1}, \breve{t}_{1}^{2}$, $\breve{t}_{2}^{1}$ and $\breve{t}_{2}^{2}, \mathcal{G}_{1}$ (without conflicts) is represented by equations: $\mathcal{G}_{1}:\left\{\begin{array}{l}x_{1}^{1}\left(k_{1}\right)=3 \otimes u_{1}\left(k_{1}\right) \oplus 3 \otimes x_{1}^{2}\left(k_{1}-1\right) \\ x_{1}^{2}\left(k_{1}\right)=5 \otimes x_{1}^{1}\left(k_{1}\right)\end{array}\right.$

Now, let's take into account the places of conflict. If the token of $\tilde{p}_{1}$ (available for the $l_{1}^{\text {th }}$ time) and the token of $\tilde{p}_{2}$ 
(available for the $l_{2}^{\text {th }}$ time) are attributed to $\mathcal{G}_{1}$ to enable its transitions to fire for the $k_{1}^{\text {th }}$ time, then we have:

$$
\left\{\begin{array}{l}
x_{1}^{1}\left(k_{1}\right)=3 \otimes u_{1}\left(k_{1}\right) \oplus 3 \otimes x_{1}^{2}\left(k_{1}-1\right) \oplus 3 \otimes \tilde{\psi}_{1}\left(l_{1}\right) \\
x_{1}^{2}\left(k_{1}\right)=5 \otimes x_{1}^{1}\left(k_{1}\right) \oplus 5 \otimes \tilde{\psi}_{2}\left(l_{2}\right) \\
\tilde{\psi}_{1}\left(l_{1}+1\right)=4 \otimes x_{1}^{1}\left(k_{1}\right), \quad \tilde{\psi}_{2}\left(l_{2}+1\right)=1 \otimes x_{1}^{2}\left(k_{1}\right)
\end{array}\right.
$$

These equations are easily brought to form (12). We let the reader write the equations relative to $\mathcal{G}_{2}$ and get to (12) with:

$$
\begin{aligned}
& A_{1}=\left(\begin{array}{ll}
\varepsilon & 3 \\
\varepsilon & 8
\end{array}\right), \quad B_{1}=\left(\begin{array}{l}
3 \\
8
\end{array}\right), \quad A_{2}=\left(\begin{array}{ll}
3 & \varepsilon \\
2 & \varepsilon
\end{array}\right), \quad B_{2}=\left(\begin{array}{l}
\varepsilon \\
\varepsilon
\end{array}\right), \\
& F_{11}=\left(\begin{array}{ll}
3 & 8
\end{array}\right)^{t}, \quad F_{12}=\left(\begin{array}{ll}
\varepsilon & 5
\end{array}\right)^{t}, \quad G_{11}=\left(\begin{array}{ll}
4 & \varepsilon
\end{array}\right), \quad G_{12}=\left(\begin{array}{ll}
\varepsilon & 1
\end{array}\right), \\
& F_{21}=\left(\begin{array}{ll}
1 & \varepsilon
\end{array}\right)^{t}, F_{22}=\left(\begin{array}{ll}
3 & 2
\end{array}\right)^{t}, G_{21}=\left(\begin{array}{ll}
4 & \varepsilon
\end{array}\right), G_{22}=\left(\begin{array}{ll}
\varepsilon & 1
\end{array}\right) .
\end{aligned}
$$

Note that all these matrices are constant and independent of resources allocation policy.

\section{LINEAR TIME-VARYING $(\mathrm{MAX},+)$ EQUATIONS OF CTEGs}

Let's recall that a linear time-varying system is of the form: $X(k)=A(k-1) \otimes X(k-1) \oplus B(k) \otimes U(k)$

This equation would be more suitable than form (12) for most problems resolution. So, one can ask question: is it possible to bring form (12) to form (15)? Answering this question is indeed crucial since modeling many existing systems using CTEGs is quite easy (e.g. the TEGs are products and the conflict places are machines) but obtaining directly (15) is not so obvious for some reasons exposed later (Remark 4.1).

\subsection{Definitions}

- D1) In practice, resources are usually allocated according to a sequence (periodic or not). So, we say that a CTEG evolves according to sequence $\sigma=\mathcal{G}_{j 1} \mathcal{G}_{j 2} \cdots \mathcal{G}_{j(k-1)} \mathcal{G}_{j k} \cdots$ (this does not sweep all admissible evolutions of CTEGs), if for each resource represented by $\tilde{p}_{j}$ and shared between two TEGs $\mathcal{G}_{j k}$ and $\mathcal{G}_{j(k+q)}$, the token of $\tilde{p}_{j}$ is first attributed to $\mathcal{G}_{j k}$ and subsequently to $\mathcal{G}_{j(k+q)}$ for all $k \geq 1, q \geq 1$. We therefore say that the $k^{\text {th }}$ realization of sequence $\sigma$ is TEG $\mathcal{G}_{i}$ if: $\mathcal{G}_{j k}=\mathcal{G}_{i}$. Example 4.1: $\tilde{R}=\left\{\tilde{p}_{1}, \tilde{p}_{2}, \tilde{p}_{3}\right\}, \tilde{R}_{1}=\left\{\tilde{p}_{1}, \tilde{p}_{3}\right\}, \tilde{R}_{2}=\left\{\tilde{p}_{2}, \tilde{p}_{3}\right\}$, $\tilde{R}_{3}=\left\{\tilde{p}_{1}, \tilde{p}_{2}\right\}, \sigma=\mathcal{G}_{1} \mathcal{G}_{2} \mathcal{G}_{2} \mathcal{G}_{1} \mathcal{G}_{3} \mathcal{G}_{3} \mathcal{G}_{3} \mathcal{G}_{2} \mathcal{G}_{1}$. According to this sequence, the token of $\tilde{p}_{1} \in\left(\tilde{R}_{1} \cap \tilde{R}_{3}\right)$ is to be attributed twice to $\mathcal{G}_{1}$ then 3 times to $\mathcal{G}_{3}$ and finally one time to $\mathcal{G}_{1}$.

- D2) Let us define the set of indices of TEGs: $S=\{1,2, \cdots, N\}$ and the function: Fre $: \tilde{R} \times S \times \mathbb{N} \mapsto S$ where Fre $\left(\tilde{p}_{j}, i, k_{i}\right)$ provides the index of the TEG that frees resource $\tilde{p}_{j}$ to be used by $\mathcal{G}_{i}$ for the $k_{i}^{\text {th }}$ time.

Example 4.1 (continued): Let's return to example 4.1 and build the following table to explain this function (a cross in a box of the row of $\tilde{p}_{j}$ and column of $\mathcal{G}_{i}$ means $\tilde{p}_{j} \in \tilde{R}_{i}$ ):

\begin{tabular}{|c|c|c|c|c|c|c|c|c|c|}
\hline$\sigma=$ & $\mathcal{G}_{1}$ & $\mathcal{G}_{2}$ & $\mathcal{G}_{2}$ & $\mathcal{G}_{1}$ & $\mathcal{G}_{3}$ & $\mathcal{G}_{3}$ & $\mathcal{G}_{3}$ & $\mathcal{G}_{2}$ & $\mathcal{G}_{1}$ \\
\hline$\tilde{p}_{1}$ & $\mathrm{x}$ & & & $\mathrm{x}$ & $\mathrm{x}$ & $\mathrm{x}$ & $\mathrm{x}$ & & $\mathrm{x}$ \\
\hline$\tilde{p}_{2}$ & & $\mathrm{x}$ & $\mathrm{x}$ & & $\mathrm{x}$ & $\mathrm{x}$ & $\mathrm{x} \longrightarrow \mathrm{x}$ & \\
\hline$\tilde{p}_{3}$ & $\mathrm{x}$ & $\mathrm{x}$ & $\longrightarrow \mathrm{x}$ & $\mathrm{x}$ & & & & $\mathrm{x}$ & $\mathrm{x}$ \\
\hline
\end{tabular}

The arrows in the table simplify the following samples: Fre $\left(\tilde{p}_{1}, 1,2\right)=1, \operatorname{Fre}\left(\tilde{p}_{2}, 2,3\right)=3$, Fre $\left(\tilde{p}_{3}, 2,2\right)=2$, and Fre $\left(\tilde{p}_{3}, 2,3\right)=1$. For instance Fre $\left(\tilde{p}_{3}, 2,3\right)=1$ means that $\mathcal{G}_{1}$ frees $\tilde{p}_{3}$ to be used by $\mathcal{G}_{2}$ for the $3^{\text {rd }}$ time.

Theorem 4.1: A CTEG verifying the hypotheses of Section 3.1 and evolving according to an arbitrary sequence $\sigma=\mathcal{G}_{j 1} \mathcal{G}_{j 2} \cdots \mathcal{G}_{j(k-1)} \mathcal{G}_{j k} \cdots$, has an equivalent Linear TimeVarying $\left(\right.$ Max, $\left.{ }^{+}\right)$representation.

Proof: Let's suppose that a CTEG verifies the hypotheses of Section 3.1 and evolves according to a sequence $\sigma$. So, we can write, from $1^{\text {st }}$ equation in (12), the following form:

$X_{i}\left(k_{i}\right)=A_{i} \otimes X_{i}\left(k_{i}-1\right) \oplus B_{i} \otimes U_{i}\left(k_{i}\right) \oplus \bigoplus_{\tilde{p}_{j} \in \tilde{R}_{i}} F_{i j} \otimes \tilde{\psi}_{j}\left(l_{j}\right)$

Also, using the $2^{\text {nd }}$ equation in (12) and function Fre we get: $\tilde{\psi}_{j}\left(l_{j}\right)=G_{\text {Fre }\left(\tilde{p}_{j}, i, k_{i}\right) j} \otimes X_{\text {Fre }\left(\tilde{p}_{j}, i, k_{i}\right)}\left(k_{\text {Fre }\left(\tilde{p}_{j}, i, k_{i}\right)}-1\right)$

So, by replacing (17) in (16), we obtain: $X_{i}\left(k_{i}\right)=A_{i} \otimes X_{i}\left(k_{i}-1\right) \oplus B_{i} \otimes U_{i}\left(k_{i}\right) \oplus$

$\bigoplus_{\tilde{p}_{j} \in \tilde{R}_{i}} F_{i j} \otimes G_{F r e\left(\tilde{p}_{j}, i, k_{i}\right) j} \otimes X_{\operatorname{Fre}\left(\tilde{p}_{j}, i, k_{i}\right)}\left(k_{\operatorname{Fre}\left(\tilde{p}_{j}, i, k_{i}\right)}-1\right)$

Let us define the $\Sigma n_{i} \times 1$ (resp. $\left.\Sigma m_{i} \times 1\right)$ vector $\bar{X}(k)$ (resp. $\bar{U}(k))$ that contains all the state vectors (resp. input vectors) of all TEGs at the $(k-1)^{\text {th }}$ realization of sequence $\sigma$ : $\bar{X}(k-1)=\left(\begin{array}{llll}X_{1}\left(k_{1}-1\right) & X_{2}\left(k_{2}-1\right) & \cdots & \left.X_{N}\left(k_{N}-1\right)\right)^{t} \quad \text { and }\end{array}\right.$ $\bar{U}(k-1)=\left(\begin{array}{llll}U_{1}\left(k_{1}-1\right) & U_{2}\left(k_{2}-1\right) & \cdots & U_{N}\left(k_{N}-1\right)\end{array}\right)^{t} \quad$ where: $k-1=\sum_{p=1}^{N}\left(k_{p}-1\right)$. If the $k^{\text {th }}$ realization of $\sigma$ is TEG $\mathcal{G}_{i}$, then we also (according to the previous definition) have: $\bar{X}(k)=\left(\begin{array}{llllll}X_{1}\left(k_{1}-1\right) & X_{2}\left(k_{2}-1\right) & \cdots & X_{i}\left(k_{i}\right) & \cdots & \left.X_{N}\left(k_{N}-1\right)\right)^{t}\end{array}\right.$ Using (18), we can therefore deduce that: $\bar{X}(k)=\bar{A}(k-1) \otimes \bar{X}(k-1) \oplus \bar{B}(k) \otimes \bar{U}(k)$ where the components of $\bar{A}(k-1)$ and $\bar{B}(k)$ are defined as: $\bar{A}(k-1)(p, q)=\left\{\begin{array}{l}I_{d} \quad \text { if }(p=q \text { and } p \neq i) \\ A_{i} \oplus \bigoplus_{\operatorname{Fre}\left(\tilde{p}_{j}, i, k_{i}\right)=i} F_{i j} \otimes G_{i j} \quad \text { if }(p=q=i) \\ \bigoplus_{\operatorname{Fre}\left(\tilde{p}_{j}, i, k_{i}\right)=q} F_{i j} \otimes G_{q j} \quad \text { if }(p=i \text { and } q \neq i) \\ I_{\varepsilon} \quad \text { otherwise }\end{array}\right.$

$\bar{B}(k)(p, q)= \begin{cases}B_{i} & \text { if }(p=q=i) \\ I_{\varepsilon} & \text { otherwise }\end{cases}$

Obviously, $(19)$ is a linear time-varying $(\mathrm{Max},+)$ system. $\square$ Note that $\bar{A}(k-1)(p, q)$ and $\bar{B}(k)(p, q)$ are not scalars but matrices. They are multiplied by the elements of $\bar{X}$ and $\bar{U}$ 
which are vectors. The $n_{p} \times n_{q}$ matrix $I_{d}$ (resp. $I_{\varepsilon}$ ) in (20) and (21) is the identity (resp. null) matrix.

Remark 4.1: It could be thought that since we can represent a system using a CTEG and subsequently equations (19), then why not do it from the beginning without passing through the CTEG modeling? Indeed, equations (19) might represent a TEG (with time-varying parameters nevertheless), equivalent to the CTEG. However, if we look closely at matrix $\bar{A}(k)$, we will notice that some of its components are, over time, sometimes null (equals $\varepsilon$ ) and sometimes not. This means literally that some places of the TEG sometimes exist and sometimes disappear. This is obviously not a usual feature with regard to Petri nets and therefore not a natural modeling of systems (see Example 5.1).

\section{5. $(\mathrm{MAX},+)$ EQUATIONS OF CYCLIC CTEGs}

Equation (19) can be obtained whatever is the CTEG evolution sequence. In practice nonetheless, many systems are repetitive and evolve according to a periodic sequence of the form $\sigma=\sigma_{0} \sigma_{0} \sigma_{0} \cdots$ where $\sigma_{0}$ is the basic sequence to be repeated. Let's suppose: $\sigma_{0}=\mathcal{G}_{j 1} \mathcal{G}_{j 2} \cdots \mathcal{G}_{j(T-1)} \mathcal{G}_{j T}$ which means that $\sigma$ is $T$-periodic. It can be easily checked that given this periodic pattern, we have:

$\operatorname{Fre}\left(\tilde{p}_{j}, i, k_{i}+T\right)=\operatorname{Fre}\left(\tilde{p}_{j}, i, k_{i}\right)$ for every triplet $\left(\tilde{p}_{j}, i, k_{i}\right)$. It follows that matrix $\bar{A}$ and $\bar{B}$ are $T$-periodic i.e. $\bar{A}(k+T)=\bar{A}(k)$ and $\bar{B}(k+T)=\bar{B}(k)$.

Hence, we can apply a well known result in conventional theory related to periodic linear systems (Bolzern et al. 1986), (Misra, 1996) and similarly used in $(\mathrm{Max},+)$ algebra (Lahaye et al. 2004). This result is a transformation of a periodic timevarying linear system into an invariant-time one using the notion of monodromy.

Let's define the transition matrix relative to matrix $\bar{A}$ as: $\Phi(i, i-q)=\left\{\begin{array}{l}\bar{A}(i-1) \otimes \bar{A}(i-2) \otimes \cdots \bar{A}(i-q) \quad \text { if } q \geq 1 \\ I_{d} \quad \text { if } q=0\end{array}\right.$

Note that $\Phi$ is $T$-periodic: $\Phi(i+T, i+T-q)=\Phi(i, i-q)$. Using (19), it follows that:

$\bar{X}(2)=\bar{A}(1) \otimes \bar{X}(1) \oplus \bar{B}(2) \otimes \bar{U}(2)$

$\bar{X}(3)=\bar{A}(2) \otimes \bar{X}(2) \oplus \bar{B}(3) \otimes \bar{U}(3)$

$=\Phi(3,1) \otimes \bar{X}(1) \oplus \Phi(3,2) \otimes \bar{B}(2) \otimes \bar{U}(2) \oplus \Phi(3,3) \otimes \bar{B}(3) \otimes \bar{U}(3)$

By recurrence, we get to:

$$
\begin{aligned}
& \bar{X}(1+T)=\Phi(1+T, 1) \otimes \bar{X}(1) \oplus \\
& \bigoplus_{q=1}^{T} \Phi(1+T, 1+q) \otimes \bar{B}(1+q) \otimes \bar{U}(1+q)
\end{aligned}
$$

Then by replacing 1 with $(1+(l-1) \cdot T)$ in (23) and using the periodicity of $\Phi$, we get to:

$$
\begin{aligned}
& \bar{X}(1+l \cdot T)=\Phi(1+T, 1) \otimes \bar{X}(1+(l-1) \cdot T) \oplus \\
& \bigoplus_{q=1}^{T} \Phi(1+T, 1+q) \otimes \bar{B}(1+q) \otimes \bar{U}(1+(l-1) \cdot T+q)
\end{aligned}
$$

Let us set $\overline{\bar{X}}(l)=\bar{X}(1+l \cdot T)$ and
$\overline{\bar{U}}(l)=(\bar{U}(1+(l-1) \cdot T+1) \cdots \bar{U}(1+(l-1) \cdot T+T))^{t}$.

Equation (24) is therefore a standard time-invariant system:

$\overline{\bar{X}}(l)=\overline{\bar{A}} \otimes \overline{\bar{X}}(l-1) \oplus \overline{\bar{B}} \otimes \overline{\bar{U}}(l)$

where $\overline{\bar{A}}=\Phi(1+T, 1)$ and

$\overline{\bar{B}}(p, q)=\left\{\begin{array}{l}\Phi(1+T, 1+q) \otimes \bar{B}(1+q) \text { if }(p=q) \\ I_{\varepsilon} \text { otherwise }\end{array}\right.$

The state matrix $\overline{\bar{A}}$ is called monodromy matrix.

Example 5.1: To illustrate how to use systematically the results above, we consider an example studied in Hillion et al. (1989) and Gaubert et al. (1999). It is a manufacturing system constituted of three machines represented by $\tilde{p}_{1}, \tilde{p}_{2}$ and $\tilde{p}_{3}$ that produce three types of parts represented by TEGs $\mathcal{G}_{1}, \mathcal{G}_{2}$ and $\mathcal{G}_{3}$ (Fig. 6). Note that: $\tilde{R}_{1}=\left\{\tilde{p}_{1}, \tilde{p}_{2}, \tilde{p}_{3}\right\}$, $\tilde{R}_{2}=\left\{\tilde{p}_{2}, \tilde{p}_{3}\right\}, \tilde{R}_{3}=\left\{\tilde{p}_{1}, \tilde{p}_{3}\right\}$ and $\tilde{\tau}_{1}=\tilde{\tau}_{2}=\tilde{\tau}_{3}=0$.

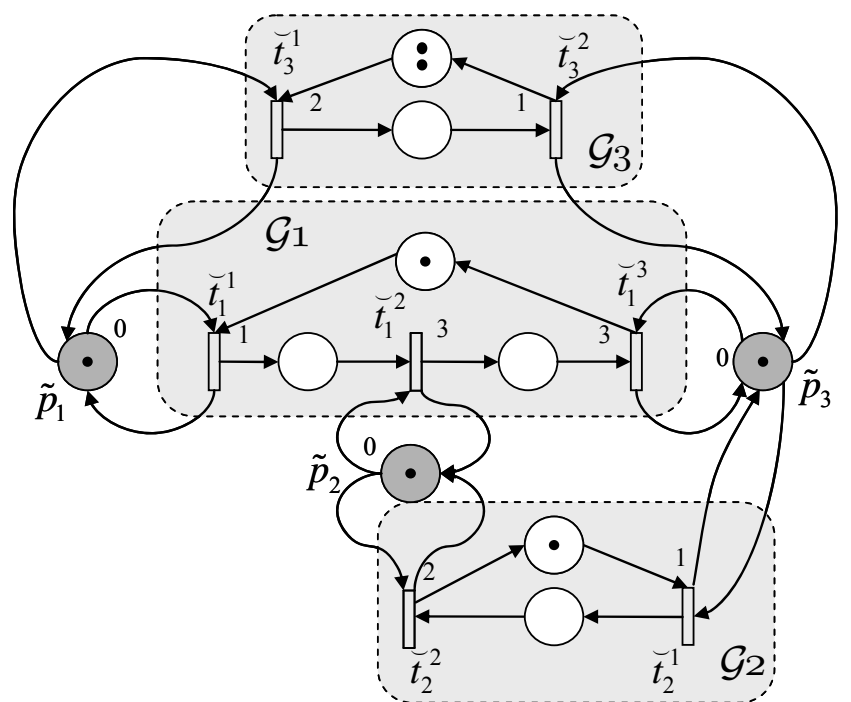

Fig. 6. A CTEG model of a manufacturing system (jobshop) involving three machines producing three types of parts.

Let us associate daters $x_{1}^{1}, x_{1}^{2}, x_{1}^{3}$ to transitions $\breve{t}_{1}^{1}, \breve{t}_{1}^{2}, \breve{t}_{1}^{3}$ and availability dates $\tilde{\psi}_{1}, \tilde{\psi}_{2}, \tilde{\psi}_{3}$ to the tokens of places $\tilde{p}_{1}$, $\tilde{p}_{2}$ and $\tilde{p}_{3}$. So, we can write:

$\left\{\begin{array}{l}x_{1}^{1}\left(k_{1}\right)=1 \otimes\left[x_{1}^{3}\left(k_{1}-1\right) \oplus \tilde{\psi}_{1}\left(l_{1}\right)\right] \\ x_{1}^{2}\left(k_{1}\right)=3 \otimes\left[\tilde{\psi}_{2}\left(l_{2}\right) \oplus x_{1}^{1}\left(k_{1}\right)\right], \\ x_{1}^{3}\left(k_{1}\right)=3 \otimes\left[\tilde{\psi}_{3}\left(l_{3}\right) \oplus x_{1}^{2}\left(k_{1}\right)\right]\end{array}\right.$,

The equations above can be brought to form (12) with:

$A_{1}=\left(\begin{array}{lll}\varepsilon & \varepsilon & 1 \\ \varepsilon & \varepsilon & 4 \\ \varepsilon & \varepsilon & 7\end{array}\right), F_{11}=\left(\begin{array}{l}1 \\ 4 \\ 7\end{array}\right), F_{12}=\left(\begin{array}{l}\varepsilon \\ 3 \\ 6\end{array}\right)$ and $F_{13}=\left(\begin{array}{l}\varepsilon \\ \varepsilon \\ 3\end{array}\right)$.

On the other hand, we have: $\tilde{\psi}_{1}\left(l_{1}+1\right)=e \otimes x_{1}^{1}(k)$, $\tilde{\psi}_{2}\left(l_{2}+1\right)=e \otimes x_{1}^{2}(k)$, and $\tilde{\psi}_{3}\left(l_{3}+1\right)=e \otimes x_{1}^{3}(k)$. So, we get: $G_{11}=\left(\begin{array}{lll}e & \varepsilon & \varepsilon\end{array}\right), G_{12}=\left(\begin{array}{lll}\varepsilon & e & \varepsilon\end{array}\right)$, and $G_{13}=\left(\begin{array}{lll}\varepsilon & \varepsilon & e\end{array}\right)$. 
We let the reader write the other equations relative to the other TEGs (add a $3^{\text {rd }}$ variable in case of $\mathcal{G}_{3}$ ) to get finally to:

$$
\begin{aligned}
& A_{2}=\left(\begin{array}{ll}
\varepsilon & 1 \\
\varepsilon & 3
\end{array}\right), \quad F_{22}=\left(\begin{array}{l}
\varepsilon \\
2
\end{array}\right), \quad F_{23}=\left(\begin{array}{l}
1 \\
3
\end{array}\right), \quad G_{22}=\left(\begin{array}{ll}
\varepsilon & e
\end{array}\right) \quad \text { and } \\
& G_{23}=\left(\begin{array}{ll}
e & \varepsilon
\end{array}\right), \quad A_{3}=\left(\begin{array}{lll}
\varepsilon & \varepsilon & 2 \\
\varepsilon & \varepsilon & 3 \\
\varepsilon & e & \varepsilon
\end{array}\right), \quad F_{31}=\left(\begin{array}{l}
2 \\
3 \\
\varepsilon
\end{array}\right), \quad F_{33}=\left(\begin{array}{l}
\varepsilon \\
1 \\
\varepsilon
\end{array}\right),
\end{aligned}
$$

$G_{31}=\left(\begin{array}{lll}e & \varepsilon & \varepsilon\end{array}\right)$ and $G_{33}=\left(\begin{array}{lll}\varepsilon & e & \varepsilon\end{array}\right)$.

All these matrices are calculated once and for all, whatever is the considered sequence. As an application, we will consider a periodic case and calculate the asymptotic production rate. Let us consider the basic sequence: $\sigma_{0}=\mathcal{G}_{1} \mathcal{G}_{2} \mathcal{G}_{3} \mathcal{G}_{3}$. We first calculate $\operatorname{Fre}\left(\tilde{p}_{j}, i, k_{i}\right)$ relative to the three resources: $\operatorname{Fre}\left(\tilde{p}_{1}, 1,1\right)=3, \operatorname{Fre}\left(\tilde{p}_{1}, 3,1\right)=1, \operatorname{Fre}\left(\tilde{p}_{1}, 3,2\right)=3, \operatorname{Fre}\left(\tilde{p}_{2}, 1,1\right)=2$, $\operatorname{Fre}\left(\tilde{p}_{2}, 2,1\right)=1, \operatorname{Fre}\left(\tilde{p}_{3}, 1,1\right)=3, \operatorname{Fre}\left(\tilde{p}_{3}, 2,1\right)=1, \operatorname{Fre}\left(\tilde{p}_{3}, 3,1\right)=2$, and $\operatorname{Pre}\left(\tilde{p}_{3}, 3,2\right)=3$. Then, matrices $\bar{A}(1), \bar{A}(2), \bar{A}(3)$ and $\bar{A}(4)$ can be calculated according to (20). They are respectively given as (without $\varepsilon$ entries):

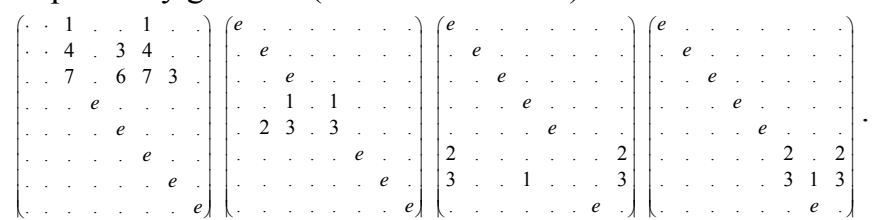

We finally calculate the monodromy matrix. It is simply

given as: $\overline{\bar{A}}=\bar{A}(4) \otimes \bar{A}(3) \otimes \bar{A}(2) \otimes \bar{A}(1)=$

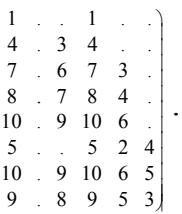

The unique finite eigenvalue of $\overline{\bar{A}}$ is equal to 9. The asymptotic production rate of $\mathcal{G}_{1}$ and $\mathcal{G}_{2}$ is therefore $\lambda_{1}=\lambda_{2}=1 / 9$ whereas the production rate of $\mathcal{G}_{3}$ is $\lambda_{3}=2 / 9$. As expected, this gives the same result as in the aforementioned works. Note however that the CTEG of Fig. 6 is not a safe Petri net and therefore the authors in Gaubert et al. (1999) used an equivalent safe one to make possible the application of the method based on heaps-of-pieces. Finding such an equivalent safe net is not always possible especially in case of presence of input transitions. The advantage of our method is also to avoid building a heap-of-pieces automaton. We can also note that the proposed approach can be applied even with infinite sequences without any effect on the CTEG structure. This is actually not the case with the method in Hillion et al. (1989) since the initial Petri net must be transformed into an equivalent TEG. Unfortunately, the resulting TEG depends dramatically on the length of the sequence and becomes very voluminous even with not long sequences (see an example in Gaubert et al. (1999)).

\section{CONCLUSION}

In this study, we extended the use of $(\mathrm{Max},+)$ algebra to Conflicting Timed Event Graphs or CTEGs which are a natural model of a large category of practical systems involving shared resources. We proved that CTEGs can be represented using a system of $(\mathrm{Max},+)$ Linear Time-Varying equations while the policy of resources allocation is arbitrary. In case of a cyclic CTEG, we used the notion of monodromy to get to a standard $(\mathrm{Max},+)$ state space representation. For future work, it would be interesting to look for the relevant modifications to bring to the proposed approach so as to relax the hypotheses of Section 3.1.

\section{REFERENCES}

Addad, B., Amari, S., and Lesage, J. J., (2010). Modélisation de réseaux de graphes d'événement temporisés avec conflits dans l'algèbre $(\mathrm{Max},+)$. IEEE Conference Internationale Francophone d'Automatique, Nancy, France, pp. (to appear).

Baccelli, F., Cohen, G., Olsder, G. J., and Quadrat, J. P., (1992). Synchronization and linearity: an algebra for discrete event systems, Wiley.

Baccelli, F., Foss, S., and Gaujal, B., (1996). Free choice Petri nets: an algebraic approach. IEEE Transactions on Automatic Control, 41(12), pp. 1751-1778.

Bolzern, P., Colaneri, P., and Scatolini, R. (1986). Zeros of discrete time linear periodic systems. IEEE Transaction on Automatic Control, vol. 31, pp. 1057-1058.

Boutin, O., Cottenceau, B., L'Anton, A., and Loiseau, J. J., (2009). Modeling systems with periodic routing functions in dioid (Min,+), 13th IFAC Symposium on Information Control Problems in Manufacturing, Moscow, Russia, pp. 1377-1382.

Cohen, G., Gaubert, S. and Quadrat, J. P., (1999). Max plus algebra and systems theory: where we are and where to go now, Annual Reviews in Control, vol. 23, pp. 207219.

Correia, A., Abbas-Turki, A., Bouyekhf, and El Moudni, A. (2009) A dioid model for invariant resource sharing problems. IEEE Trans. on Systems, Man, and Cybernetics-Part A: Systems and Humans, 39(4), pp. 770-781.

Gaubert, S., and Mairesse, J., (1999). Modeling and analysis of timed Petri nets using heaps of pieces. IEEE Transactions on Automatic Control, 44(4), pp. 683-697.

Hillion, H. and Proth, J. M., (1989). Performance evaluation of jobshop systems using timed event graphs, IEEE Transaction on Automatic Control, 34(1), pp. 3-9.

Lahaye, S., Boimond, J. L., and Hardouin, L., (2004) Linear periodic systems over dioids, Discrete Event Dynamic Systems, 14(2), pp 133-152.

Misra, P., (1996). Time-Invariant representation of discrete periodic systems, Automatica, 32(2), pp. 267-272.

Murata, T., (1989). Petri nets: properties analysis and applications. Proc of the IEEE, 77(4), pp. 541-580.

Nait, S. M., Manier, M. A., El Moudni, A., and Wack, M. (2006). Petri net with conflicts and (max,plus) algebra for transportation systems, 11th IFAC Symposium on Transportation Systems, The Netherlands.

Van Den Boom, T. J. J, and De Shutter, B., (2006). Modeling and control of discrete event systems using switching Max-Plus linear systems, Control Engineering Practice, 14(10), pp. 1199-1211. 